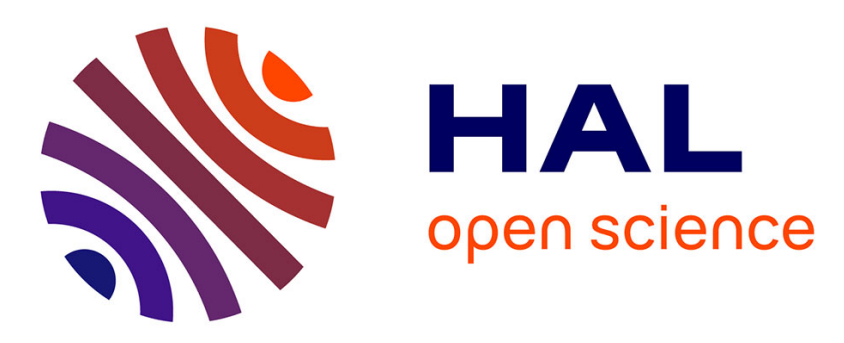

\title{
Photosensitized oxidative addition to gold(i) enables alkynylative cyclization of o-alkylnylphenols with iodoalkynes
}

Zhonghua Xia, Vincent Corcé, Fen Zhao, Cédric Przybylski, Agathe Espagne, Ludovic Jullien, Thomas Le Saux, Yves Gimbert, Héloïse Dossmann, Virginie Mouriès-Mansuy, et al.

\section{To cite this version:}

Zhonghua Xia, Vincent Corcé, Fen Zhao, Cédric Przybylski, Agathe Espagne, et al.. Photosensitized oxidative addition to gold(i) enables alkynylative cyclization of o-alkylnylphenols with iodoalkynes. Nature Chemistry, 2019, 11 (9), pp.797-805. 10.1038/s41557-019-0295-9 . hal-02282216

\section{HAL Id: hal-02282216 https://hal.sorbonne-universite.fr/hal-02282216}

Submitted on 22 Oct 2020

HAL is a multi-disciplinary open access archive for the deposit and dissemination of scientific research documents, whether they are published or not. The documents may come from teaching and research institutions in France or abroad, or from public or private research centers.
L'archive ouverte pluridisciplinaire HAL, est destinée au dépôt et à la diffusion de documents scientifiques de niveau recherche, publiés ou non, émanant des établissements d'enseignement et de recherche français ou étrangers, des laboratoires publics ou privés. 


\title{
Photosensitized oxidative addition to gold(I) enables alkynylative cyclization of $o$ - alkylnylphenols with iodoalkynes
}

Zhonghua Xia ${ }^{1}$, Vincent Corcé ${ }^{1}$, Fen Zhao ${ }^{1}$, Cédric Przybylski ${ }^{1}$, Agathe Espagne ${ }^{2}$, Ludovic Jullien ${ }^{2}$, Thomas Le Saux ${ }^{2}$, Yves Gimbert ${ }^{1,3}$, Héloïse Dossmann ${ }^{1}$, Virginie Mouriès-Mansuy ${ }^{*}$, Cyril Ollivi$\mathrm{er}^{1 *}$ and Louis Fensterbank ${ }^{1 *}$

${ }^{1}$ Sorbonne Université, CNRS, Institut Parisien de Chimie Moléculaire, IPCM, 4 place Jussieu, F75005 Paris, France

${ }^{2}$ PASTEUR, Département de chimie, École normale supérieure, PSL University, Sorbonne Université, CNRS, 24, rue Lhomond, 75005 Paris, France

${ }^{3}$ Université Grenoble Alpes and CNRS, DCM (UMR 5250), CS 40700 - 38058 Grenoble Cedex 9 , France

\begin{abstract}
The well-established oxidative addition - reductive elimination pathway is the most followed one in transition metal catalyzed cross coupling reactions. While readily occurring with a series of transition metals, it does not take place with gold(I) complexes which have shown some reluctance to undergo oxidative addition unless special sets of ligands on gold(I), reagents or reaction conditions are used. A new possibility to overcome this hurdle has been devised. Upon visible light irradiation, an iridium photocatalyst triggers via triplet sensitization the oxidative addition of an alkynyliodide onto a vinylgold(I) intermediate to deliver $\operatorname{Cs} p^{2}-\mathrm{Cs} p$ coupling products after reductive elimination. Mechanistic and modeling studies support that an energy transfer takes place and not a redox pathway. This novel mode of activation in gold homogenous catalysis was applied in several dual catalytic processes. Alkynylbenzofuran derivatives were obtained from $o$-alkynylphenols and iodoalkynes in the presence of catalytic gold(I) and iridium(III) complexes under blue LED irradiation.
\end{abstract}

Over the last two decades, homogeneous gold catalysis has been extensively used to efficiently and selectively promote a variety of cyclization processes. ${ }^{1-3}$ The typical casting involves bifunctional substrates bearing an unsaturation prompt to electrophilic activation and a judiciously positioned internal nucleophile. A protodemetalation of the organogold intermediates to afford hydrofunctionalized products generally terminates the catalytic cycles. ${ }^{4}$ Pursuing the step economy principle and also aiming at higher level of molecular complexity, some in situ post-functionalization reactions of the organogold ${ }^{5}$ intermediate have been devised such as electrophilic halogenation or cross-coupling reactions. Although palladium catalyzed cross coupling from an organogold(I) intermediate has been 
rendered possible, ${ }^{6,7}$ this transformation still needs generality and most of the described coupling reactions have transited through a $\mathrm{Au}(\mathrm{I})$ to $\mathrm{Au}(\mathrm{III})$ oxidation necessitating an oxidant in stoichiometric quantity, transmetalation and reductive elimination cycle. ${ }^{8,9}$ A notable breakthrough in this area was achieved by Glorius ${ }^{10,11}$ and Toste ${ }^{12,13}$ who bypassed the burden of stoichiometric oxidants by merging gold catalysis with photoredox catalysis which ensures the oxidation states shuttle. ${ }^{14-16}$ Arylative cyclization and related transformations as well as cross coupling processes have been devised relying on the use of easily reduced aryl diazonium salts (Fig. 1a). ${ }^{17-24}$ Two mechanism pathways have been proposed (Fig. 1b) which both feature the reductive elimination from a vinylgold(III) intermediate of type D. But they differ by the stage of addition of the radical on gold (intermediate A vs F). Pathway I has been recently supported by stoichiometric reactions and calculations. ${ }^{9,25,26}$ A groundbreaking advance in these reactions would be to promote the oxidative addition step by energy transfer (photosensitization) as it has found more and more relevance in visible light catalysis involving also organometallic complexes. ${ }^{27}$ Useful photophysical guidelines about Dexter vs. Förster and exergonic vs. endergonic energy transfers have been drawn recently for the synthetic chemist. $^{28}$

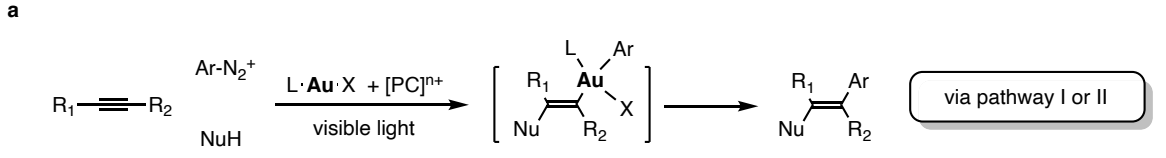

b

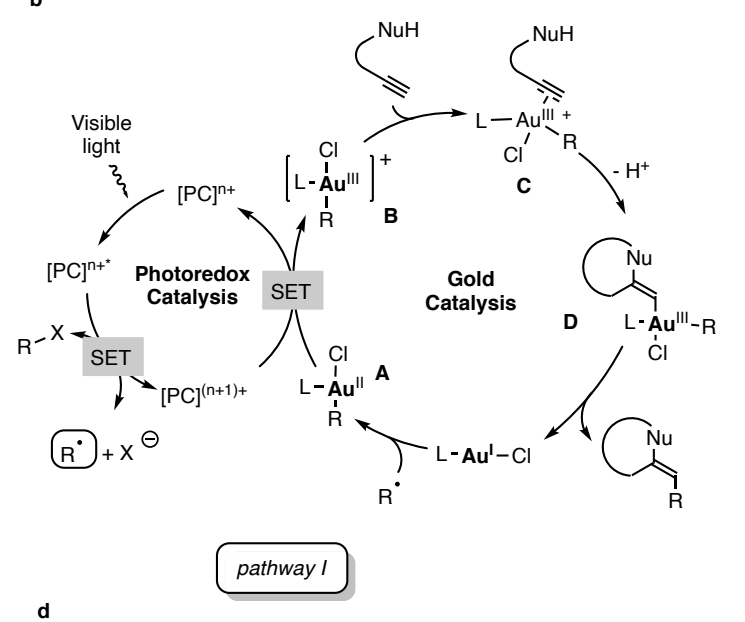

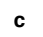

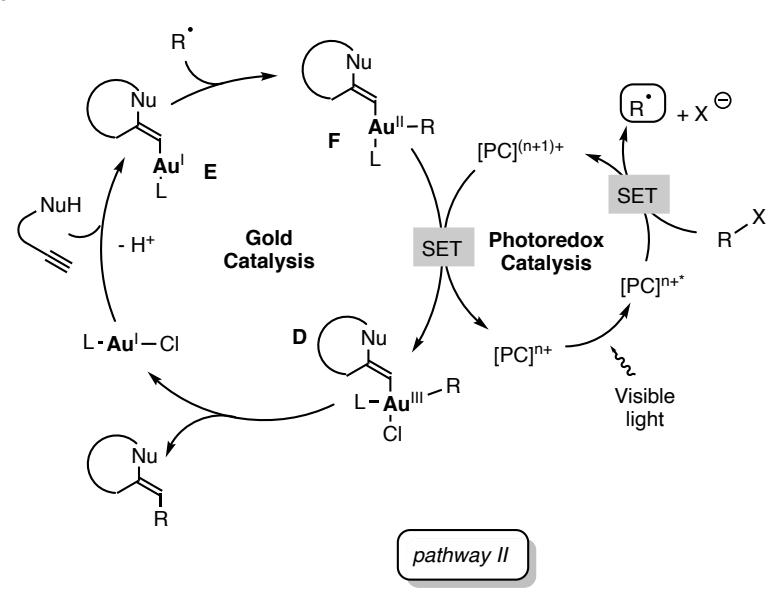

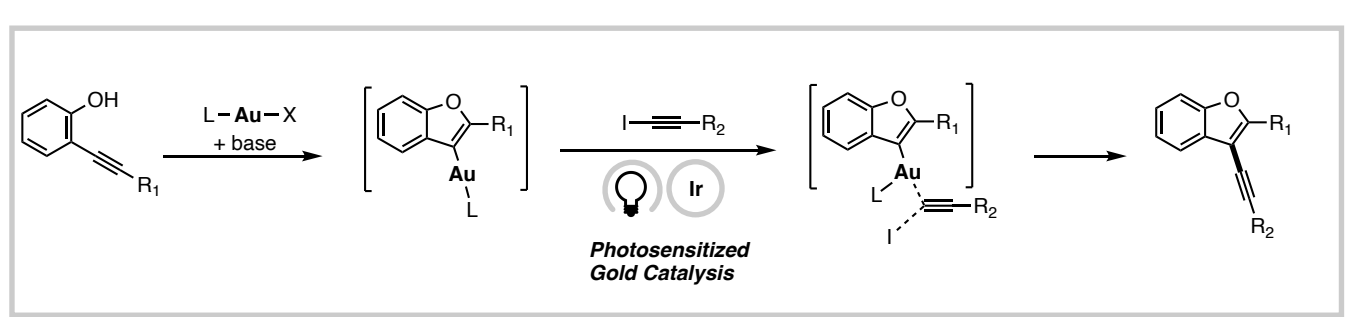

Figure 1 
Extending the scope of possible partners in these transformations has also appeared highly desirable and we aimed at developing some novel alkynylative cyclization processes corresponding to a formal Csp $p^{2}$-Csp cross-coupling reaction which has little precedent in this type of dual catalytic transformations. ${ }^{13,29-31}$ Thus, replacing aryl diazoniums by alkynyl iodide partners in our recent dual photoredox/gold catalyzed arylative cyclization of $o$-alkynylphenols which leads to benzofurans, ${ }^{32}$ would constitute an appropriate ground of exploration as well as provide valuable scaffolds. We were also aware that alkynyl iodides are much less reactive than aryl diazoniums and that we would probably have to devise a distinct mode of activation of gold(I) complexes in order to promote the C-C bond formation step (Fig. 1c). Indeed, gold(I) complexes are notoriously reluctant to oxidative additions. ${ }^{33}$ It can be rendered feasible only by using special sets of electrophilic reagents ${ }^{34-36}$ and/or conditions. For instance, it was shown by Toste that $\mathrm{CF}_{3} \mathrm{I}$ adds to arylgold(I) complexes under UV irradiation. ${ }^{37}$ Substrates bearing a directing group or with inherent ring $\operatorname{strain}^{38,39}$ can also undergo oxidative addition to provide cyclometallated gold(III) intermediates. Recently also, Amgoune and Bourissou, ${ }^{40,41}$ and Russell, ${ }^{42}$ have demonstrated that bidentate ligands on gold(I) with particular features promote oxidative addition and that the resulting gold(III) intermediate can react with a nucleophile to provide cross coupling products, ${ }^{42}$ notably through a catalytic cycle. ${ }^{41}$ In this work, we uncover a completely novel mode of $\mathrm{C}-\mathrm{C}$ bond formation via photosensitized energy transfer which promotes oxidative addition at a gold(I) complex (Fig. 1c).

\section{Results}

Optimization studies. We rapidly surveyed the feasibility of such a transformation by examining the model reaction between 2-( $p$-tolyl-ethynyl)phenol 1a and iodoethynyl benzene $\mathbf{2 a}$ under various conditions (see Supplementary section III for detailed conditions optimization). Preliminary negative results based on the previous arylation protocol using a catalytic mixture of $\mathrm{Ru}(\mathrm{bpy})_{3} \mathrm{Cl}_{2}$ and $\mathrm{PPh}_{3} \mathrm{AuCl}$ in $\mathrm{MeOH}$ drove us to other conditions. Notably, first hits in the formation of benzofuran 3aa (structure confirmed by X-ray diffraction analysis, CCDC 1850903) were obtained in acetonitrile and by adding a base (see Supplementary section III.2 and Table 1, entry 1). This finding was consolidated by using $\operatorname{Ir}\left[\mathrm{dF}\left(\mathrm{CF}_{3}\right) \mathrm{ppy}\right]_{2}(\mathrm{dtbbpy}) \mathrm{PF}_{6}([\mathbf{I r}-\mathbf{F}])$ as photocatalyst (entry 3, 26\% of 3aa). A substantial gain of yield was observed (56\% of 3aa) by switching $\mathrm{PPh}_{3} \mathrm{AuCl}$ to $\left(p-\mathrm{CF}_{3} \mathrm{Ph}\right)_{3} \mathrm{PAuCl}$ [Au-CF $\mathbf{A}]$ (entry 4). Finally, after substantial optimization it was found that the combination of [Au$\left.\mathbf{C F}_{3}\right]$ (5 mol \%), ([Ir-F]) (1 mol\%), 1,10-phenanthroline (10 mol\%), $\mathrm{K}_{2} \mathrm{CO}_{3}$ (2.5 equiv) in degassed $\mathrm{MeCN}$ at r.t. overnight under blue LED light gave the best result since a $71 \%$ isolated yield of 3aa was obtained (entry 5). Interestingly, the reaction can work without photocatalyst (entry 8) and the use of a more reductive photocatalyst such as $f a c$ - $\operatorname{Ir}(\mathrm{ppy})_{3}$ was not rewarding (entry 2). Control ex- 
periments regarding the role of 1,10-phenanthroline were also performed. A stoichiometric amount of 1,10-phenanthroline proved detrimental to the yield (entry 6), however other amines such as quinuclidine (entry 7) or TMEDA, DABCO, DBU (see Supplementary section III.4) could also be used to improve the reaction. In sharp contrast, no desired product could be obtained in the absence of base $\left(\mathrm{K}_{2} \mathrm{CO}_{3}\right.$, entry 11$)$, gold catalyst $\left(p-\mathrm{CF}_{3} \mathrm{Ph}\right)_{3} \mathrm{PAuCl}$ (entry 10), or light (entry 12). Finally, it is worthy of note that iodoetherification product $\mathbf{4 a}$ and diyne $\mathbf{5 a}$ were the side compounds in almost all conditions. Diyne 5a was present in lower quantity $(<20 \%)$ and its formation presumably requires gold catalysis (see Supplementary section III.6). Moreover, when (bromoethynyl)benzene 2aBr was subjected to the reaction, only $9 \%$ of 3 aa was obtained.

Mechanistic investigations. This preliminary set of findings drove us to delineate a plausible mechanism for further development of the reaction. We first considered the addition of a radical intermediate stemming from the photocatalytic cycle to produce the corresponding intermediate of type $\mathbf{B}$ through A (Fig. 1). Alkynyl radicals indeed remain elusive species but they have been mentioned sporadically in the literature to be generated from alkynyl iodides. ${ }^{43,44}$ Nevertheless, by using alkynyl iodide $\mathbf{2 b}$ as a probe because it bears a fluorine label, this hypothesis was rapidly discarded. First, as the reductive potential of 1-fluoro-4-(iodoethynyl)benzene $\left(E_{1 / 2}(\mathbf{2 b})=-1.47 \mathrm{~V} v s \mathrm{SCE}\right)$ is significantly higher than $[\mathbf{I r}-\mathbf{F}]$ catalyst $\left(E^{*}{ }_{1 / 2}=-0.89 \mathrm{~V} v_{S} \mathrm{SCE}\right)$, photoreductive formation of an alkynyl radical appeared quite unlikely. This was corroborated by fluorescence quenching studies which showed no quench of excited $[\mathbf{I r}-\mathbf{F}]\left({ }^{3} \mathrm{~T}_{1}\right)$, noted ${ }^{3}[\mathbf{I r}-\mathbf{F}]$, by $\mathbf{2} \mathbf{b}$ therefore precluding a photocatalyzed electron transfer event. Another pathway for the formation of the gold(III) intermediate of type $\mathbf{B}$ was investigated based on the fact that alkynyl iodide $\mathbf{2 b}$ and gold complex [Au-CF $\mathbf{C}$ ] convert into a new unstable gold species under blue LED irradiation but the catalytic role of the latter remains elusive (see Supplementary section V.5).

Based on all literature reports ${ }^{9,14,16}$ a vinyl gold(III) intermediate of type D of Fig. 1 which would undergo reductive elimination to provide benzofurans $\mathbf{3}$ is presumably involved. To investigate on this, vinylgold(I) 6 of type $\mathbf{E}$ was considered and prepared in $82 \%$ yield by an independent route ${ }^{45}$ as shown in Fig. 2a. X-ray diffraction analysis of suitable crystals of $\mathbf{6}$ confirmed it structure (CCDC 1850902, Fig. 2b) and provided useful structural data for further modeling studies. When $\mathbf{6}$ was confronted to one equivalent of alkynyl iodide $\mathbf{2 b}$, no conversion was observed after 3 hours at room temperature $\left(25^{\circ} \mathrm{C}\right)$. However, blue LED irradiation changed the scenario. Indeed, a low conversion $(<10 \%)$ was observed after $2 \mathrm{~h}$ at room temperature while overnight irradiation resulted in the formation 3ab in 33\% (accompanied by $20 \%$ of $\mathbf{4 a}$ and $35 \%$ of protodeauration product 7 as determined by ${ }^{1} \mathrm{H}$ NMR (Fig. 2c). Addition of $10 \mathrm{~mol} \%$ of [Ir-F] dramatically altered the outcome and 
yielded benzofuran 3ab almost quantitatively (Fig. 2d). Therefore, experimental conditions to trigger the key $\mathrm{C}-\mathrm{C}$ bond formation were successfully found. Although, the benzofuran formation could be achieved under direct irradiation conditions without photocatalyst [Ir-F] (Figs. 2c \& 2d) which brings some rationalization for the finding of entries 8 and 9 of Table 1, it appears highly enhanced in its presence. This was confirmed by measuring the steady-state luminescence spectra of mixtures of $[\mathbf{I r}-\mathbf{F}]$ and 6. We observed a drop of the [Ir-F] luminescence signal upon increasing the concentration of $\mathbf{6}$ which suggested that $\mathbf{6}$ acts as a quencher of the triplet state ${ }^{3} \mathrm{~T}_{1}$ of $[\mathbf{I r}-\mathbf{F}]\left({ }^{3}[\mathbf{I r}-\mathbf{F}]\right)$ (Fig. 2e). In parallel, we recorded the luminescence lifetime of the same solutions and observed that it decreased from $2.4 \mu \mathrm{s}$ in the absence of 6 (in agreement with previous reports ${ }^{46,47}$ ) to $290 \mathrm{~ns}$ in the presence of $745 \mu \mathrm{M}$ of $\mathbf{6}$, thereby confirming the quenching of ${ }^{3}[\mathbf{I r}-\mathbf{F}]$ by $\mathbf{6}$ (see Supplementary Fig. 3). Values of $2.9 \times 10^{9} \mathrm{~mol}^{-1} \cdot \mathrm{L} \cdot \mathrm{s}^{-1}$ and $4.1 \times 10^{9} \mathrm{~mol}^{-1} \cdot \mathrm{L} \cdot \mathrm{s}^{-1}$ were extracted for the $k_{q}$ bimolecular quenching rate constant from respectively the luminescence intensity $\left(\mathrm{I}_{0} / \mathrm{I}\right)$ and lifetime $\left(\tau_{0} / \tau\right)$ SternVolmer plots (see inset of Fig. 2e and Supplementary Fig. 4). These $k_{q}$ values are almost in the range of the encounter rate under control of molecular diffusion. This observation suggests that no major molecular reorganization occurs during the reaction between ${ }^{3}[\mathbf{I r}-\mathbf{F}]$ and $\mathbf{6}$, which would be reasonably in line with a Dexter type energy transfer according to (Eq. 1):

${ }^{3}[\mathrm{Ir}-\mathrm{F}]+\mathbf{6} \rightarrow[\mathrm{Ir}-\mathrm{F}]+{ }^{\mathbf{3}} \mathbf{6}^{28}$

while considering that the triplet level of ${ }^{\mathbf{3}}[\mathbf{I r}-\mathbf{F}]$ is considerably higher than that of ${ }^{\mathbf{3}} \mathbf{6} .{ }^{48}$

We also recorded the transient absorption spectra of [ $\mathbf{I r}-\mathbf{F}]$ solutions containing various concentrations of 6. Fig. $2 \mathrm{f}$ displays the results. The differential spectrum of ${ }^{3}[\mathbf{I r}-\mathbf{F}]$ exhibits a maximum around 480-500 nm, which is in fair agreement with the literature. ${ }^{49}$ The addition of an equimolar amount of $\mathbf{6}$ yields a decrease of the ${ }^{3}[\mathbf{I r}-\mathbf{F}]$ signal consistent with the ${ }^{3}[\mathbf{I r}-\mathbf{F}]$ luminescence quenching observed in Fig. 2e. In the presence of a nine-fold excess of $\mathbf{6}$, the ${ }^{3}[\mathbf{I r}-\mathbf{F}]$ signal almost disappears and it is replaced by a broad differential absorption tentatively attributed to ${ }^{\mathbf{3}} \mathbf{6}$ (new contributions below $450 \mathrm{~nm}$ and above $550 \mathrm{~nm}$ ).

Of note also, the formation of 3ab is enhanced by the presence of $10 \mathrm{~mol} \%$ benzophenone in the reaction medium which presumably also acts as a sensitizer (Fig. 2d).

Another important point to check was the formation of $\mathbf{6}$ in the reaction conditions. This was achieved by exposing phenol 1a to a stoichiometric amount of [Au-CF $\mathbf{A}]$ in $\mathrm{CD}_{3} \mathrm{CN}$ overnight. After such reaction time in the dark, the formation of vinygold(I) 6 was observed by NMR in $26 \%$ yield (See Supplementary section V.4).

All the previous studies converge toward the possible implication of an excited state of $\mathbf{6}$ whose formation is promoted by the long-lived triplet state ${ }^{3}[\mathbf{I r}-\mathbf{F}] .{ }^{50}$ This finding also bears some resonance with a recent report on "excited-state organometallic catalysis" by McCusker and MacMil- 
lan, ${ }^{51}$ who reported an energy-transfer mediated reductive elimination on an excited arylnickel(II) intermediate and in the same vein as a few related reports on copper ${ }^{52,53}$ and other nickel intermediates, ${ }^{54-56}$ Further support to these conclusions was brought by calculations. The spin density of ${ }^{\mathbf{3}}$ [IrF] was indeed compared either isolated or in the vicinity of 6 (Fig. $2 \mathrm{~g}$ and $2 \mathrm{~h}$ and Supplementary sections VIII.2-3 for calculation details). As shown on Fig. 2h, part of the ${ }^{3}[\mathbf{I r}-\mathbf{F}]$ spin density is transferred to the approaching furan moiety of the gold complex $\mathbf{6}$, intimating that energy transfer is taking place. This would lead to the formation of $\mathbf{6}$ in an excited electronic state which may further react with iodoethynyl benzene $\mathbf{2 a}$. Note that the same calculations performed on ${ }^{\mathbf{3}}[\mathbf{I r}-\mathbf{F}]$ approached by $2 \mathbf{a}$ show that no transfer is occurring on 2a (see Supplementary Fig.7). This finding is consistent with the quenching studies done on $[\mathbf{I r}-\mathbf{F}]$ in the presence of $\mathbf{6}$ or $\mathbf{2 a}$. Note also that precatalyst [Au$\mathbf{C F}_{3}$ ] does not quench the fluorescence of [Ir-F]. To determine which electronic states of $\mathbf{6}$ are accessible via this energy transfer, time-dependent DFT (TD-DFT) calculations were carried out. Results show that only the ${ }^{3} \mathrm{~T}_{1}$ excited state 6 is accessible within the blue LED energy range (470 $\mathrm{nm}$, see Supplementary section VIII.3 for details). 

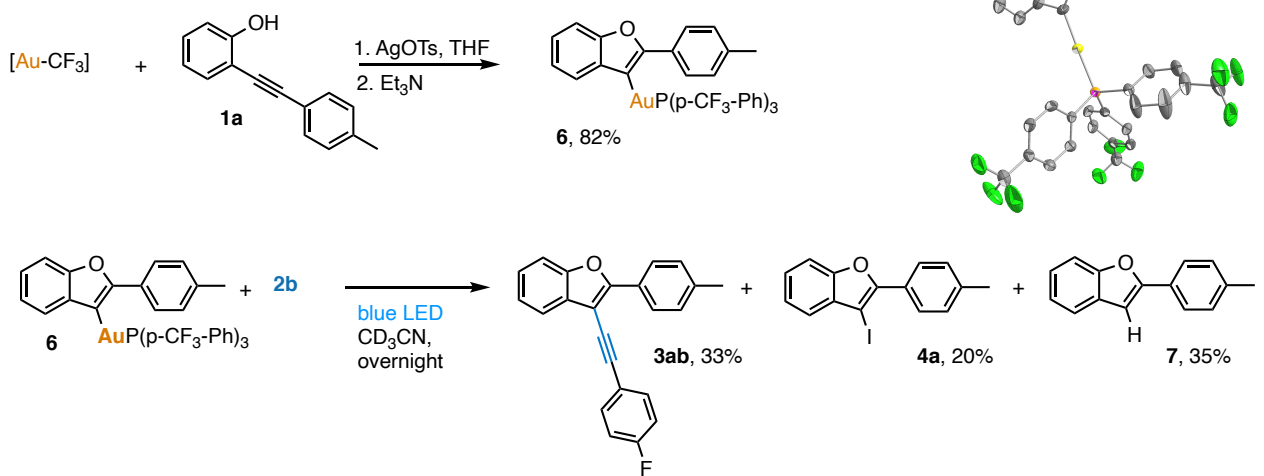

d
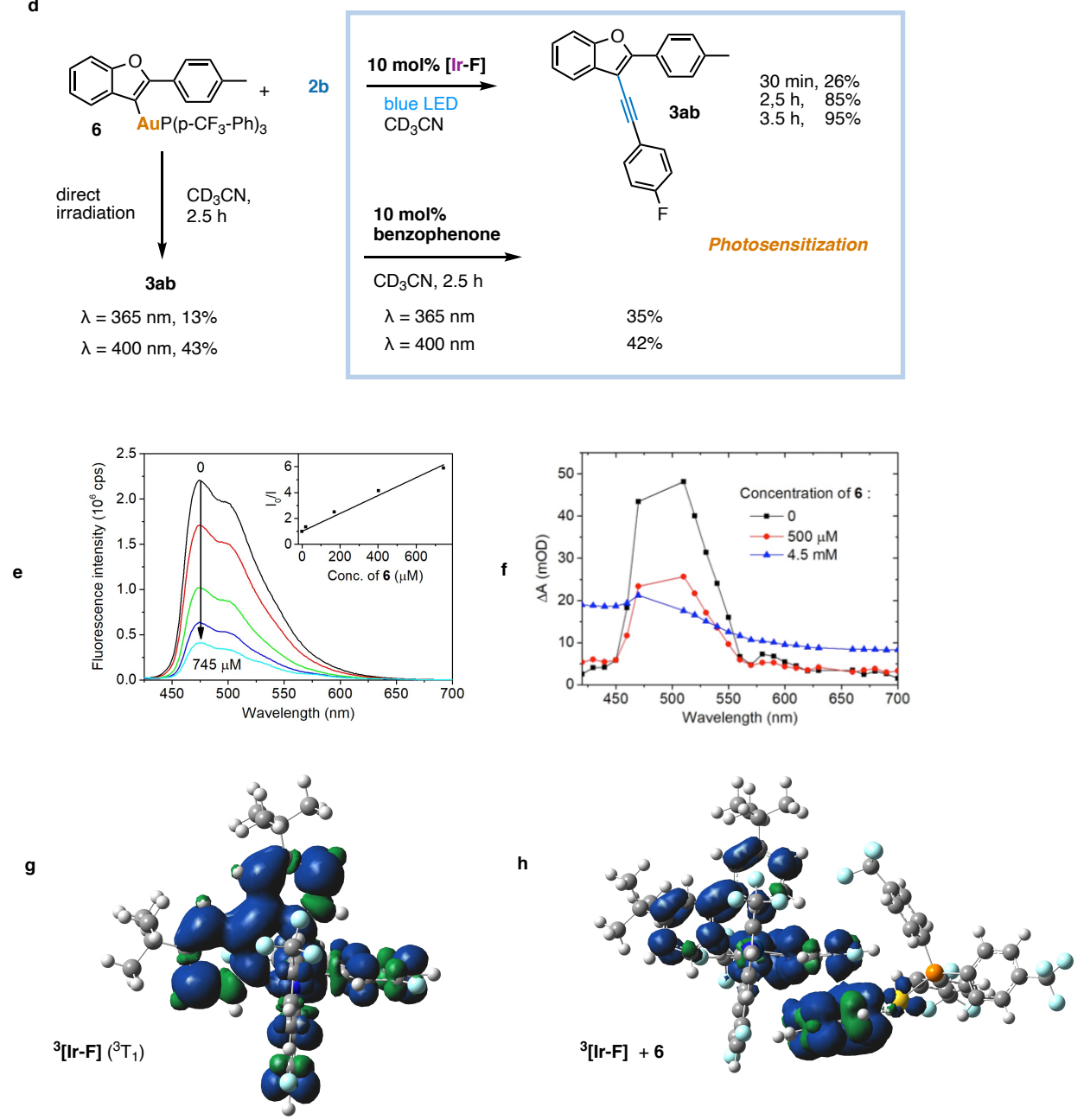

Figure 2

Modeling studies. Following these findings, we have therefore conducted a detailed theoretical study of the reaction of $\mathbf{6}$ with $\mathbf{2 a}$ with $\mathbf{6}$ in its ${ }^{3} \mathrm{~T}_{1}$ excited state. The ground state reactivity of $\mathbf{6}$ was also checked in order to help rationalizing the role of blue LED in the mechanism efficiency. For the sake of clarity, we will now refer to $\mathbf{6}$ either as ${ }^{\mathbf{1}} \mathbf{6}$ or as ${ }^{\mathbf{3}} \mathbf{6}$ to unambiguously refer to the ${ }^{1} \mathrm{~S}_{0}$ ground state or the ${ }^{3} \mathrm{~T}_{1}$ excited state of $\mathbf{6}$, respectively. All calculations presented below were obtained at the 
PBE0/SDD(Au), 6-311G*(I), 6-31G** (other atoms) level of theory, taking into account solvent effects by SMD procedure. If not stated otherwise, reported energies are $\Delta \mathrm{G}_{\mathrm{MeCN}}$.

On the singlet potential energy surface, the reaction pathway was quite straightforward to determine. However the barrier of formation of the oxidative addition adduct, gold(III) complex ${ }^{\mathbf{1}} \mathbf{I}$ is prohibitive: almost $28 \mathrm{kcal} / \mathrm{mol}$ (see Supplementary section VIII.4) and renders the overall pathway unlikely. On the contrary, the reaction on the triplet potential energy surface appeared more sinuous but more favorable thermodynamically (see Fig. 3). The approach of $\mathbf{2 a}$ to ${ }^{\mathbf{3}} \mathbf{6}$ (along the Au-C (bearing the iodine) bond reaction coordinate) is leading to the formation of an intermediate complex ${ }^{3} \mathbf{I I}$, lying down $18.5 \mathrm{kcal} / \mathrm{mol}$ below the reagents. Interestingly, the geometry adopted by $\mathbf{2 a}$ in this complex is bent (I-C-C angle of $115^{\circ}$ ) and reminiscent of that of modeled ${ }^{3} \mathbf{2 a}$ (I-C-C angle of $129^{\circ} v S$ $180^{\circ}$ for $\mathbf{2 a}$ in its ground state, see Supplementary Fig.15). This feature suggests that the ${ }^{\mathbf{3}} \mathbf{6}$ complex may transfer energy to $\mathbf{2 a}$ when these reactants are approaching each other. Checking the spin density along the Au-C (bearing the iodine) bond reaction coordinate effectively confirms that a transfer is occurring at a relatively long distance (from $3.6 \AA$, see Supplementary Fig.16). Therefore, ${ }^{\mathbf{3} 6}$ could act as a relay for transferring energy to $\mathbf{2 a}$ which would provide access to a reactive bent structure of the acetylenic compound. From complex ${ }^{3} \mathbf{I I}$ and by approaching the iodine atom to gold, intermediate ${ }^{3} \mathbf{I I I}$ is localized on which $\mathrm{Au}(\mathrm{I})$ is oxidized to $\mathrm{Au}(\mathrm{III})$ and organic precursors lie in trans position. This step occurs with a low activation energy of $2.2 \mathrm{kcal} / \mathrm{mol}$ via TS3. By reducing the $\mathrm{C}$-Au-C angle on ${ }^{3}$ III (i.e. bringing together the two $\mathrm{C}$ atoms involved in the forthcoming new $\mathrm{C}$ $\mathrm{C}$ bond), it was possible to localize a transition structure TS4 requiring a formation barrier of 18.8 $\mathrm{kcal} / \mathrm{mol}$. TS4 connects to the Au(III) complex intermediate ${ }^{3} \mathbf{I V}$ on which the formation of the key $\mathrm{C}-\mathrm{C}$ bond between $\mathbf{6}$ and $\mathbf{2 a}$ is observed but with the iodine still interacting with the slightly elongated triple bond $(\mathrm{d}(\mathrm{I}-\mathrm{C})=2.29 \AA)$. Finally, from ${ }^{3} \mathbf{I V}$, two pathway variants can be envisaged. First, the ${ }^{3} \mathbf{I V}$ complex may further rearrange to lead to a ${ }^{3} \mathbf{V}$ complex $\left(-27 \mathrm{kcal} / \mathrm{mol}\right.$ below $\left.{ }^{3} \mathbf{I V}\right)$ via an inexpensive TS5 transition structure $\left(+0.001 \mathrm{kcal} / \mathrm{mol}\right.$ compared to $\left.{ }^{3} \mathbf{I V}\right)$. Then ${ }^{3} \mathbf{V}$ easily dissociates to lead to ${ }^{\mathbf{3}} \mathbf{3} \mathbf{a a}+\left(p-\mathrm{CF}_{3} \mathrm{Ph}\right)_{3} \mathrm{PAuI}$ (barrier TS6 of $\left.6 \mathrm{kcal} / \mathrm{mol}\right)$. An electronic decay of ${ }^{\mathbf{3}} \mathbf{3} \mathbf{a a}$ can then be envisaged to lead to the final coupling product $3 \mathbf{a a}+\left(p-\mathrm{CF}_{3} \mathrm{Ph}\right)_{3} \mathrm{PAuI}$. Another possible pathway would imply a direct $\mathrm{S}_{0} \leftarrow \mathrm{T}_{1}$ electronic decay of ${ }^{3} \mathbf{I V}$, leading to a complex which appears to be dissociative. It would thus give directly the cited above final coupling products. 


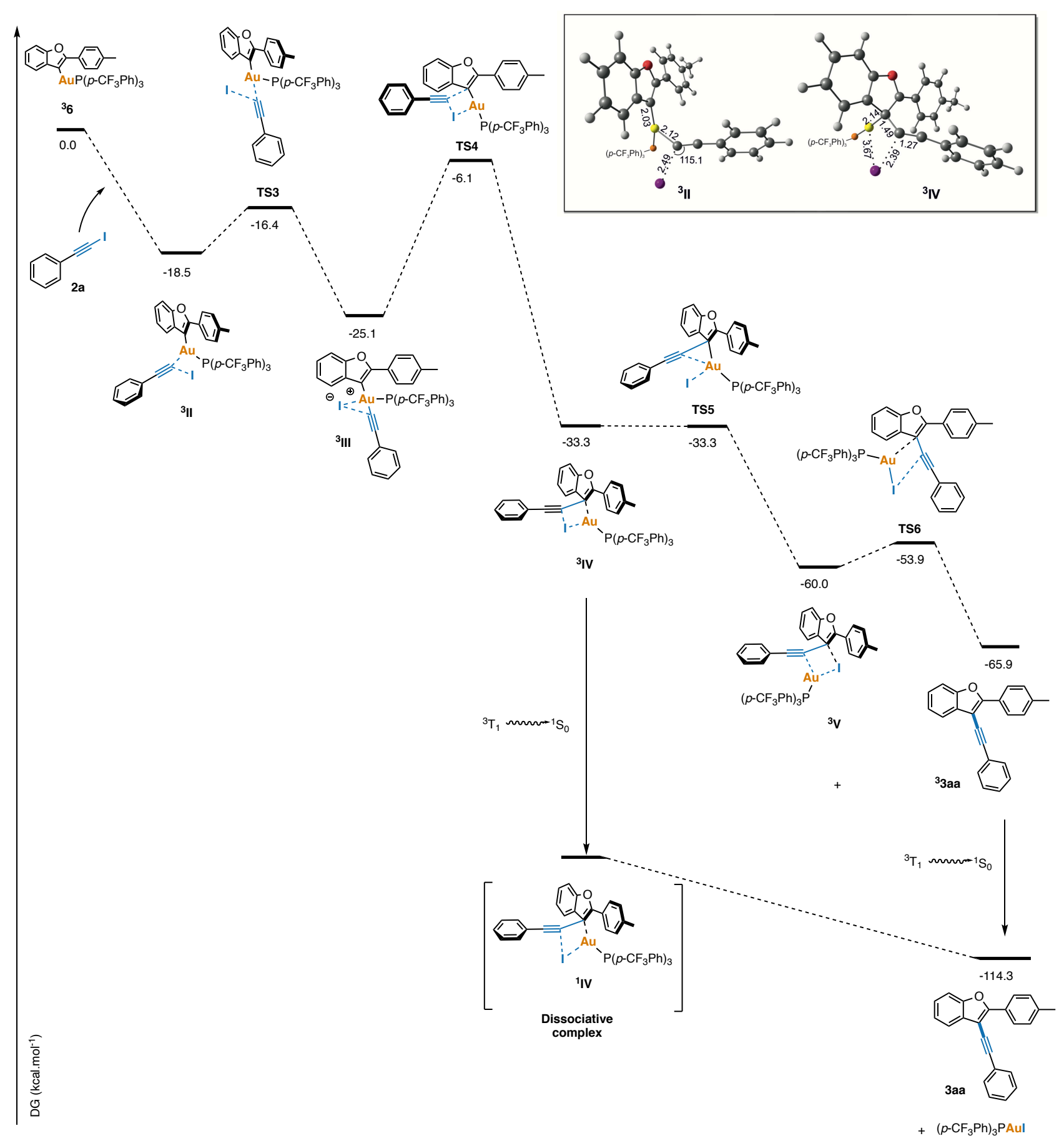

Figure 3

The proposed reaction pathway using ${ }^{\mathbf{3}} \mathbf{6}$ as a starting product appears thus extremely favorable from a thermodynamic point of view, since all intermediate complexes and transition structures have indeed lower energies than the starting products. This would be highly coherent with the great efficiency of the observed reaction of Fig. 2d. Altogether, the aforementioned data support the plausible catalytic pathway of Fig. 4. 
In addition to the use of the photosensitizer [Ir-F], two additional factors appear to optimize this process. First, the substitution of $\mathrm{Ph}_{3} \mathrm{PAuCl}$ by $\left[\mathbf{A u}-\mathbf{C F}_{3}\right.$ ] appeared highly beneficial (Table 1, entry 3 vs 4), maybe due to the higher electrophilicity of $\left[\mathbf{A u}-\mathbf{C F}_{3}\right] \cdot{ }^{25}$ Second, the adjunction of 1,10phenanthroline allowed to observe a significant yield increase whatever the followed pathway. Compare for instance entry 4 vs 5 of Table 1 or entry $8 v s 9$ of Table 1 . The reason for this is not clearly established and several hypotheses are standing. For instance, some halogen bonding between phenanthroline and the alkynyl iodides $\mathbf{2}$, which are known halogen bonding donors, ${ }^{57}$ might be at play and explain the increased reactivity of the system.

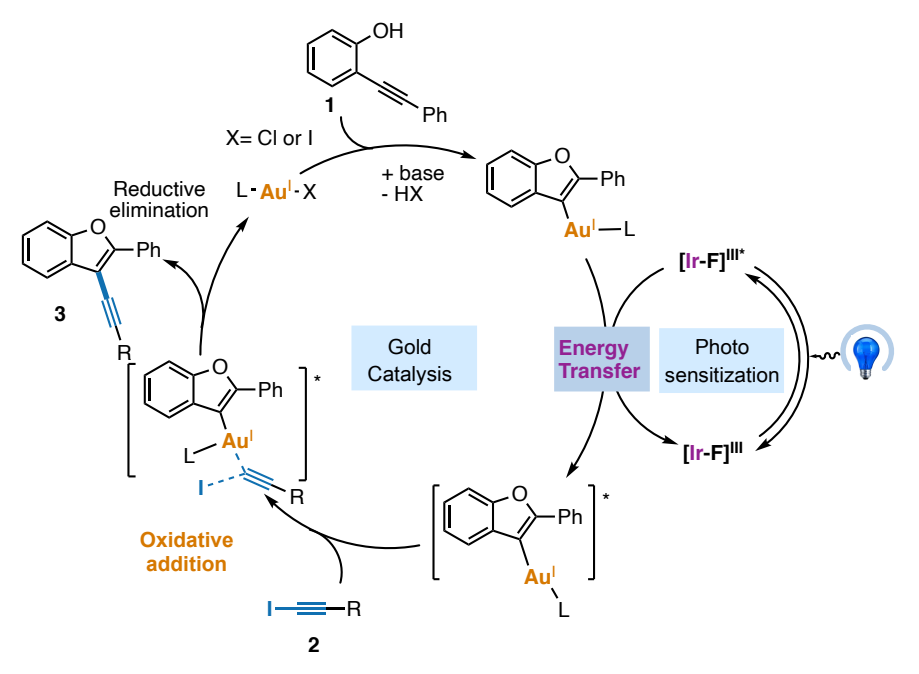

Figure 4

Scope of the reaction. We then explored the scope of this transformation. For this, a series of substituted aryl iodoalkynes $\mathbf{2}$ were reacted with 2-(phenylethynyl)phenols 1 under the optimized reaction conditions (Fig. 5a). Aryl iodoethynyl bearing electron-withdrawing groups in the para position, such as $\mathrm{F}$ and $\mathrm{CF}_{3}$ afforded corresponding benzofurans 3ab and 3ac in good yields. Meta $\mathrm{F}$ or ortho $\mathrm{Cl}$ substituted aryl iodoalkynes reacted smoothly with 2-(phenylethynyl)phenol 1a, providing 3ad and 3ae in $78 \%$ and $63 \%$ yields, respectively. The presence of electron donating groups (-Me, $-t$ - Bu, $-\mathrm{OMe})$ at the para position or no substitution furnished the alkynylbenzofurans in slightly lower (3af, 3ag and 3ba) to moderate (3ah) yields. No reaction was observed with an iodoalkyne bearing a 4-nitro arylgroup, instead the protodeauration cyclization product 7 was obtained. In addition, alkylalkynes bearing $n$-pentyl, cyclohexyl, and 3-nitrile butyl groups could be incorporated into the benzofurans scaffold (3aj-3al) from the corresponding alkyl iodoalkynes, albeit in significantly lower yields (25-42\% yield). 


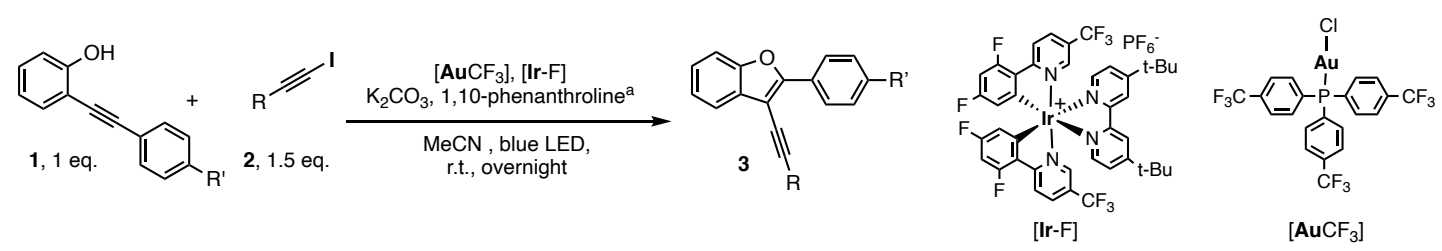

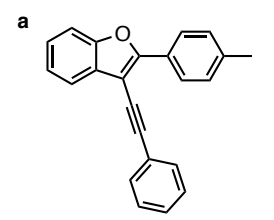

3aa, $71 \%$

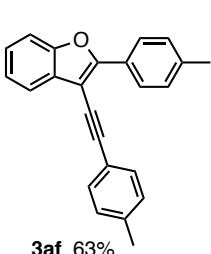

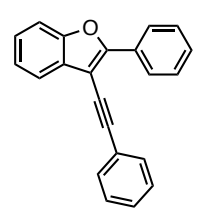

3ba, $67 \%$
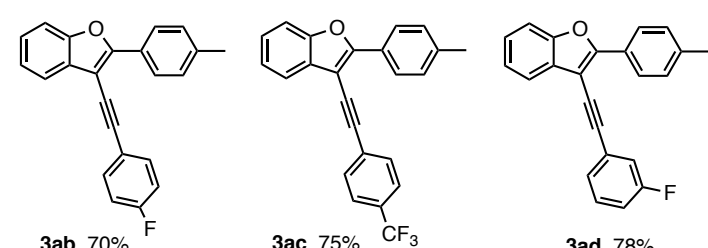

3ad, $78 \%$

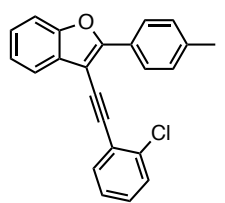

3ae, $63 \%$
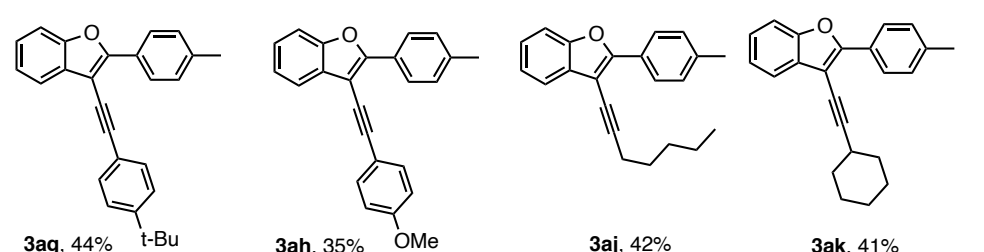

3ak, $41 \%$

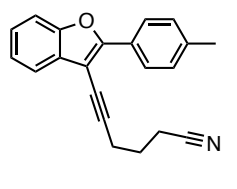

3aj, $42 \%$

3al, 25\%
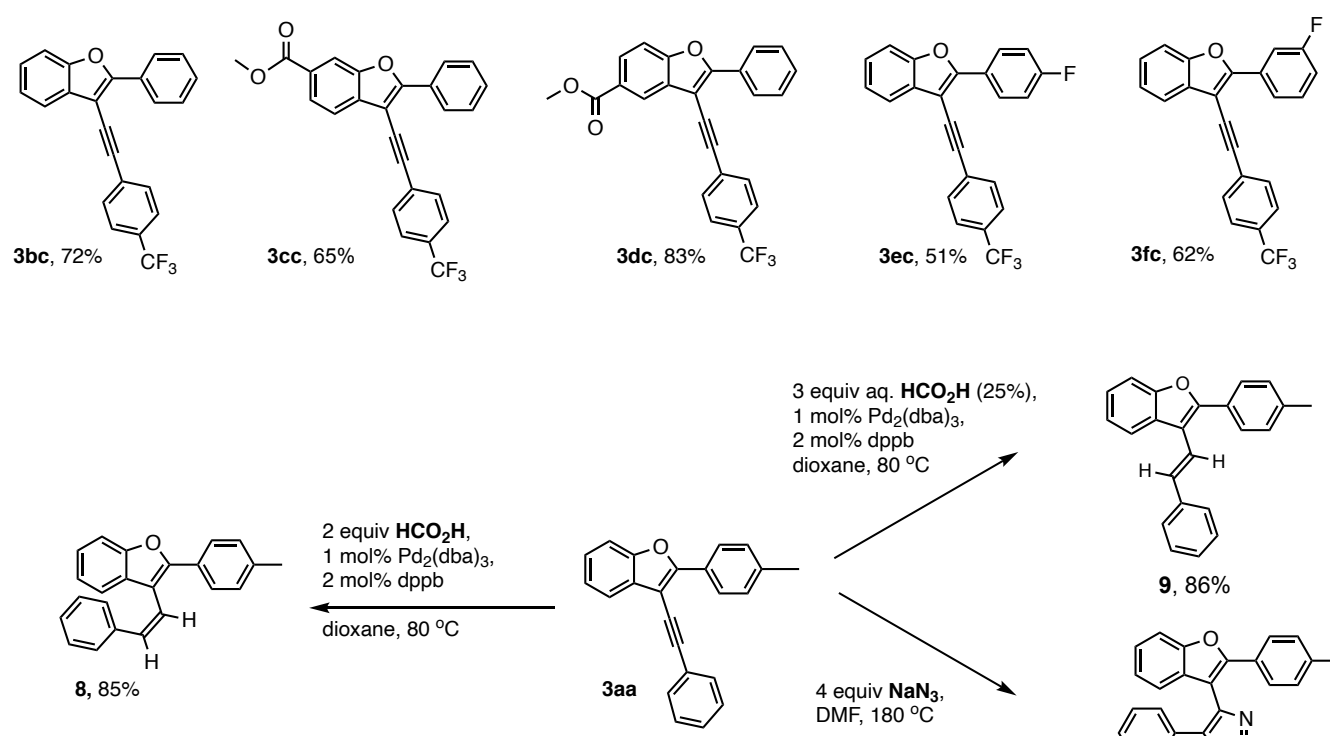

d

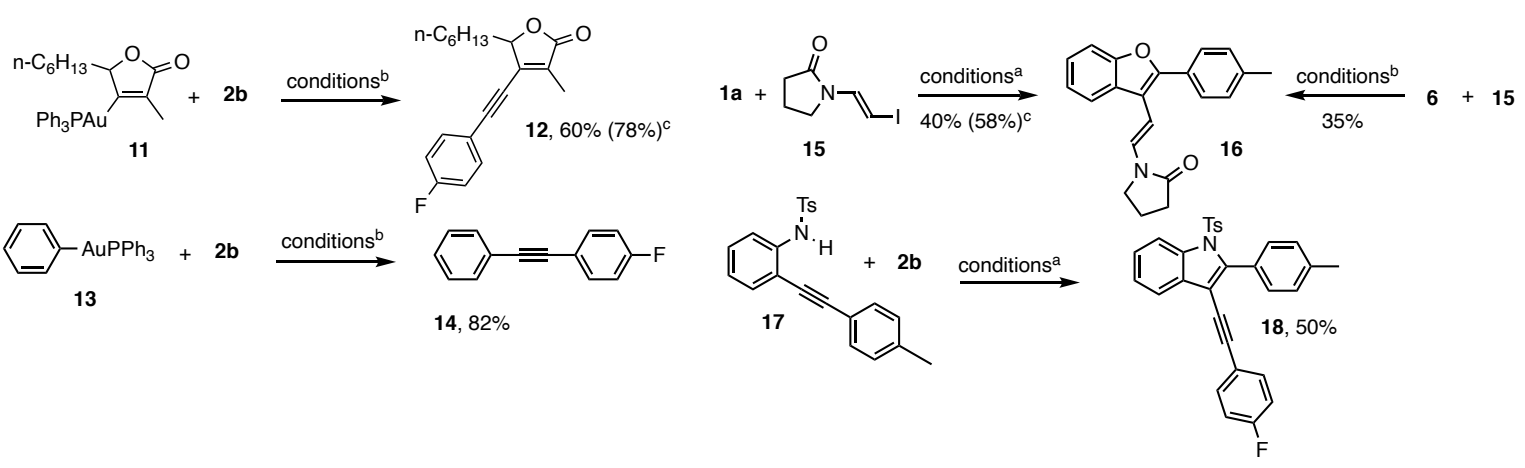

Figure 5

Fig. 5. a Iodoalkynes 2 scope. b $O$-alkynylphenols 1 scope. c Post-functionalization of benzofuran 3aa. d Other working systems. 
The effect of substitution on both aromatic rings of $o$-alkynylphenols 1 was then investigated in reactions with 1-(iodoethynyl)-4-(trifluoromethyl)benzene 2c (Fig. 5b). Arylalkynes with no substitution or bearing a $\mathrm{CH}_{3}, \mathrm{~F}$ group at the para or meta position to the alkyne gave good yields of benzofurans (3bc,3ac, 3ec and 3fc). Similarly, precursors with an electron-withdrawing ester group on the phenol moiety at the para or meta position of the alkyne, delivered the corresponding ethynylbenzofurans in good yields (3cc and 3dc).

Postfunctionalization and other systems. The benzofurans 3 are valuable scaffolds for further elaboration notably through the potential reactivity of the alkyne moiety. For instance, the product 3aa can be hydrogenated by formic acid under palladium(0) catalysis. Depending on the reaction conditions $Z$-alkene $\mathbf{8}$ or the $E$-isomer $\mathbf{9}$ can be selectively obtained. Triazole $\mathbf{1 0}$ could also be formed in thermal conditions through a Huisgen type of reaction between 3aa and sodium azide (Fig. 5c, see Supplementary section VI for details).

Finally, the sensitization protocol is not restricted to substrates $\mathbf{1}$ and $\mathbf{2}$. Other organogold intermediates such as vinylgold $\mathbf{1 1}$ and arylgold $\mathbf{1 3}$ can undergo the oxidative addition - reductive elimination sequence to provide respectively $\mathbf{1 2}$ and $\mathbf{1 4}$ as shown in Fig. 5d. One pot-reactions are also possible using a vinyliodide electrophile (15) or an $o$-alkynyl tosylaniline (17) as nucleophilic precursor. In the latter case, a new route to 2,3-disubstituted indoles is open.

\section{Conclusions}

This study brings in light a new type of dual catalysis type of transformation involving electrophilic gold catalysis and iridium photosensitization to allow a Csp2-Csp cross coupling reaction useful for the alkynylation of benzofurans. Thanks to a thorough luminescence study supported by DFT calculations, a novel mechanistic pathway has been uncovered. Blue LED excited $\operatorname{Ir}\left[\mathrm{dF}\left(\mathrm{CF}_{3}\right) \text { ppy }\right]_{2}(\mathrm{dtbbpy}) \mathrm{PF}_{6}$ indeed interacts with a vinylgold(I) intermediate stemming from a gold(I) promoted 5-endo-dig $O$-cyclization via energy transfer to trigger oxidative addition at gold(I). In other words, the triplet excited state of the vinylgold(I) intermediate and the alkynyl iodide partner readily engages in an oxidative addition -trans/cis isomerization sequence which forges the desired Csp2-Csp bond and after desexciting reductive elimination delivers the benzofuran product. This completely undescribed process in gold catalysis tunnels the difficult oxidative addition reductive elimination sequence and, since other nucleophilic and electrophilic partners are competent, it opens new avenues in the field of excited state gold catalysis

\section{Methods}

General Procedure: Alkynative Cyclization of $o$-Alkynylphenols with Iodoalkynes. 
To a Schlenk tube equipped with a magnetic stirring bar and charged with the photocatalyst $\operatorname{Ir}\left[d \mathrm{~F}\left(\mathrm{CF}_{3}\right) \text { ppy }\right]_{2}(\mathrm{dtbbpy}) \mathrm{PF}_{6}(1 \mathrm{~mol} \%)$, the gold(I) complex $\left(p-\mathrm{CF}_{3} \mathrm{Ph}\right)_{3} \mathrm{PAuCl}(5 \mathrm{~mol} \%), \mathrm{K}_{2} \mathrm{CO}_{3}$ (2.5 equiv), phenanthroline $(10 \mathrm{~mol} \%)$, the appropriate iodoalkyne $2(0.15 \mathrm{mmol})$ and $o$ alkynylphenol derivative $1(0.1 \mathrm{mmol})$ was introduced $\mathrm{MeCN}(2 \mathrm{~mL})$. The mixture was degassed using three freeze pump-thaw cycles and purged with Ar, then irradiated for $16 \mathrm{~h}$ (unless mentioned) with blue LED light (see Supplementary section IV for setup). The stirring speed was equal to or more than $1200 \mathrm{rpm}$. The reaction was quenched with $\mathrm{Et}_{2} \mathrm{O}(3 \mathrm{~mL})$ and a $2 \mathrm{M} \mathrm{HCl}$ solution $(3 \mathrm{~mL})$ and the solution was extracted by $\mathrm{Et}_{2} \mathrm{O}(3 \times 5 \mathrm{~mL})$. The combined organic layer was dried over $\mathrm{MgSO}_{4}$, filtered and concentrated under reduced pressure to give the crude product. The residue was purified by FC on silica gel to afford the desired benzofuran product $\mathbf{3}$.

Data availability. Crystallographic data for the structures reported in this Article have been deposited at the Cambridge Crystallographic Data Centre, under deposition numbers CCDC 1850903 (3aa) and 1850902 (6). Copies of the data can be obtained free of charge via https://www.ccdc.cam.ac.uk/structures/. All other data supporting the findings of this study are available within the Article and its Supplementary Information, or from the corresponding authors upon reasonable request.

\section{REFERENCES}

(1) Dorel, R. \& Echavarren A. M. Gold(I)-catalyzed activation of alkynes for the construction of molecular complexity. Chem. Rev. 115, 9028-9072 (2015).

(2) Fensterbank, L. \& Malacria, M. Molecular complexity from polyunsaturated substrates: the gold catalysis approach. Acc. Chem. Res. 47, 953-965 (2014).

(3) Harris, R. J. \& Widenhoefer, R. A. Gold carbenes, gold-stabilized carbocations, and cationic intermediates relevant to gold-catalysed enyne cycloaddition. Chem. Soc. Rev. 45, 4533-4551 (2016).

(4) Wang, W., Hammond, G. B. \& Xu, B. Ligand effects and ligand design in homogeneous gold(I) catalysis. J. Am. Chem. Soc. 134, 5697-5705 (2012).

(5) Liu, L.-P. \& Hammond, G. Recent advances in the isolation and reactivity of organogold complexes. Chem. Soc. Rev. 41, 3129-3139 (2012).

(6) Hashmi, A. S. K. et al. Scope and limitations of palladium-catalyzed cross-coupling reactions with organogold compounds. Adv. Synth. Catal. 352, 1307-1314 (2010).

(7) Garcia-Dominguez, P. \& Nevado, C. Au-Pd Bimetallic Catalysis: The importance of anionic ligands in catalyst speciation. J. Am. Chem. Soc. 138, 3266-3269 (2016).

(8) Zheng, Z., Wang, Z., Wang, Y. \& Zhang, L. Au-Catalysed oxidative cyclisation. Chem. Soc. Rev. 45, 4448-4458 (2016). 
(9) Hopkinson, M. N., Tlahuext-Aca, A. \& Glorius, F. Merging visible light photoredox and gold catalysis. Acc. Chem. Res. 49, 2261-2272 (2016).

(10) Sahoo, B., Hopkinson, M. N. \& Glorius, F. Combining gold and photoredox catalysis: visible light-mediated oxy- and aminoarylation of alkenes. J. Am. Chem. Soc. 135, 5505-5508 (2013).

(11) Tlahuext-Aca, A., Hopkinson, M. N., Sahoo, B. \& Glorius, F. Dual gold/photoredox-catalyzed $\mathrm{C}(s p)-\mathrm{H}$ arylation of terminal alkynes with diazonium salts. Chem. Sci. 7, 89-93 (2016).

(12) Shu, X.-Z., Zhang, M., Frei, H. \& Toste, F. D. Dual visible light photoredox and gold-catalyzed arylative ring expansion. J. Am. Chem. Soc. 136, 5844-5847 (2014).

(13) For a dual catalysis example on a different system (aryldiazonium - TMS-alkyne), see: Kim, S., Rojas-Martin, J. \& Toste, F. D. Visible light-mediated gold-catalysed carbon( $s p 2)$-carbon( $s p)$ crosscoupling. Chem. Sci. 7, 85-88 (2016).

(14) For reviews, see: Levin, M. D.; Kim, S. \& Toste, F. D. Photoredox catalysis unlocks singleelectron elementary steps in transition metal catalyzed cross-coupling. ACS Cent. Sci. 2, 293-301 (2016).

(15) Skubi, K. L., Blum, T. R. \& Yoon, T. P. Dual catalysis strategies in photochemical synthesis. Chem. Rev. 116, 10035-10074 (2016).

(16) Twilton, J., Le, C., Zhang, P., Evans R. W. \& MacMillan, D. W. C. The merger of transition metal and photocatalysis. Nat. Rev. Chem. 1, 0052 (2017).

(17) Patil, D. V., Yun, H. \& Shin, S. Catalytic cross-coupling of vinyl golds with diazonium salts under photoredox and thermal conditions. Adv. Synth. Catal. 357, 2622-2628 (2015).

(18) Um, J., Yun, H. \& Shin, S. Cross-coupling of Meyer-Schuster intermediates under dual goldphotoredox catalysis. Org. Lett. 18, 484-487 (2016).

(19) Huang, L., Rudolph, M., Rominger, F. \& Hashmi, A. S. K. Photosensitizer-free visible light mediated gold catalyzed 1,2-difunctionalization of alkynes. Angew. Chem. Int. Ed. 55, 4808-4813 (2016).

(20) Tlahuext-Aca, A., Hopkinson, M. N., Garza-Sanchez, R. A., \& Glorius, F. Alkyne difunctionalization by dual gold/photoredox catalysis. Chem. Eur. J. 22, 5909-5913 (2016).

(21) Alcaide, B., Almendros, P., Busto, E. \& Luna, A. Domino Meyer-Schuster/arylation reaction of alkynols or alkynyl hydroperoxides with diazonium salts promoted by visible light under dual gold and ruthenium catalysis. Adv. Synth. Catal. 358, 1526-1533 (2016).

(22) Cornilleau, T., Hermange, P. \& Fouquet, E. Gold-catalysed cross-coupling between aryldiazonium salts and arylboronic acids: probing the usefulness of photoredox conditions. Chem. Commun. 52, 10040-10043 (2016).

(23) Gauchot, V. \& Lee, A.-L. Dual gold photoredox C(sp2)-C(sp2) cross couplings - development and mechanistic studies. Chem. Commun. 52, 10163-10166 (2016). 
(24) Gauchot, V., Sutherland, D. R. \& Lee, A.-L. Dual gold and photoredox catalysed C-H activation of arenes for aryl-aryl cross couplings. Chem. Sci. 8, 2885-2889 (2017).

(25) Tlahuext-Aca, A., Hopkinson, M. N., Daniliuc, C. G. \& Glorius, F. Oxidative addition to gold(I) by photoredox catalysis: straightforward access to diverse $(C, N)$ - cyclometalated gold(III) complexes. Chem. Eur. J. 22, 11587-11592 (2016).

(26) Zhang, Q., Zhang, Z.-Q., Fu, Y. \& Yu, H.-Z. Mechanism of the visible light-mediated goldcatalyzed oxyarylation reaction of alkenes. ACS Catal. 6, 798-808 (2016).

(27) Zhou, Q.-Q.; Zou, Y.-Q.; Lu, L.-Q. \& Xiao, W.-J. Visible-light-induced organic photochemical reactions through energy-transfer pathways. Angew. Chem. Int. Ed. 58, 1586-1604 (2019).

(28) Strieth-Kalthoff, F.; James, J. M.; Teders, M.; Pitzer, L.\& Glorius, F. Energy transfer catalysis mediated by visible light: principles, applications, directions. Chem. Soc. Rev. 47, 7190-7202 (2018).

(29) For a seminal gold catalyzed alkynylation of arenes relying on an external stoichiometric oxidant, see: de Haro, T. \& Nevado, C. Gold-Catatalyzed Ethynylation of Arenes. J. Am. Chem. Soc. 132, 1512-1513 (2010).

(30) For a seminal gold catalyzed alkynylation of a vinylgold intermediate relying on an external stoichiometric oxidant, see: Hopkinson, M. N., Ross, J. E., Giuffredi, G. T., Gee, A. D. \& Gouverneur, V. Gold-catalyzed cascade cyclization-oxidative alkynylation of allenoates. Org. Lett. 12, 4904-4907 (2010).

(31) For a gold-catalyzed alkynylation using alkynyl-benziodoxolones, see: Li, Y., Brand, J. P. \& Waser, J. Gold-catalyzed regioselective synthesis of 2- and 3-alkynyl furans. Angew. Chem. Int. Ed. 52, 6743-6747 (2013).

(32) Xia, Z., Khaled, O., Mouriès-Mansuy, V., Ollivier, C. \& Fensterbank, L. Dual photoredox/gold catalysis arylative cyclization of o-alkynylphenols with aryldiazonium salts: a flexible synthesis of benzofurans. J. Org. Chem. 81, 7182-7190 (2016).

(33) For a review, see: Joost, M., Amgoune, A. \& Bourissou, D. Reactivity of gold complexes towards elementary organometallic reactions. Angew. Chem. Int. Ed. 54, 15022-15045 (2015).

(34) Levin, M. D. \& Toste, F. D. Gold-catalyzed allylation of aryl boronic acids: accessing crosscoupling reactivity with gold. Angew. Chem. Int. Ed. 53, 6211-6215 (2014).

(35) Asomoza-Solis, E. O., Rojas-Ocampo, J., Toscano, R. A. \& Porcel, S. Arenediazonium salts as electrophiles for the oxidative addition of gold(I). Chem. Commun. 52, 7295-7298 (2016).

(36) Huang, L., Rominger, F., Rudolph, M. \& Hashmi, A. S. K. A General access to organogold(III) complexes by oxidative addition of diazonium salts. Chem. Commun. 52, 6435-6438 (2016).

(37) Winston, M. S., Wolf, W. J. \& Toste, F. D. Photoinitiated oxidative addition of $\mathrm{CF}_{3} \mathrm{I}$ to gold(I) and facile aryl-cf 3 reductive elimination. J. Am. Chem. Soc. 136, 7777-7782 (2014).

(38) Serra, J., Parella, T. \& Ribas, X. Au(III)-Aryl intermediates in oxidant-free C-N and C-O crosscoupling catalysis. Chem. Sci. 8, 946-952 (2017).

(39) Joost, M. et al. Oxidative addition of carbon-carbon bonds to gold. Angew. Chem. Int. Ed. 54, 14512-14516 (2015). 
(40) Joost, M. et al. Facile oxidative addition of aryl iodides to gold(I) by ligand design: bending turns on reactivity. J. Am. Chem. Soc. 136, 14654-14657 (2014).

(41) Zeineddine, A. et al. Rational development of catalytic $\mathrm{Au}(\mathrm{I}) / \mathrm{Au}(\mathrm{III})$ arylation involving mild oxidative addition of aryl

halides. Nat. Comm. 8, 565 (2017).

(42) Harper, M. J. et al. Oxidative addition, transmetalation, and reductive elimination at a 2,2bipyridyl-ligated gold center J. Am. Chem. Soc. 140, 4440-4445 (2018).

(43) Martelli, G., Spagnolo, P. \& Tiecco, M. Homolytic aromatic substitution by phenylethynyl radicals. J. Chem. Soc. (B) 0, 1413-1418 (1970).

(44) Xie, J. et al. A Highly efficient gold-catalyzed photoredox $\alpha-\mathrm{C}(s p 3)-\mathrm{H}$ alkynylation of tertiary aliphatic amines with sunlight. Angew. Chem. Int. Ed. 54, 6046-6050 (2015).

(45) Hashmi, A. S. K., Ramamurthi, T. D. \& Rominger, F. On the trapping of vinylgold intermediates. Adv. Synth. Catal. 352, 971-975 (2010).

(46) M. S. Lowry et al. Single-layer electroluminescent devices and photoinduced hydrogen production from an ionic iridium(III) complex. Chem. Mater. 17, 5712-5719 (2005).

(47) M. Teders et al. The energy-transfer-enabled biocompatible disulfide-ene reaction. Nat. Chem.10, 981-988 (2018).

(48) G. Porter, F. Wilkinson, Energy transfer from the triplet state, Proc. Roy. Soc. A, 1961, 264, 118.

(49) A. Kaga et al. Degenerative xanthate transfer to olefins under visible-light photocatalysis. Beilstein J. Org. Chem. 2018, 14, 3047-3058.

(50) For an energy transfer with [Ir-F], see: Lu, Z. \& Yoon, T. P. Visible light photocatalysis of [2+2] styrene cycloadditions by energy transfer. Angew. Chem. Int. Ed. 51, 10329-10332 (2012).

(51) Welin, E. R., Le, C., Arias-Rotondo, D. M., McCusker, J. K. \& MacMillan, D. W. C. Photosensitized energy transfer-mediated organometallic catalysis through electronically excited nickel(II). Science 355, 380-385 (2017).

(52) For a seminal report with direct irradiation, see: Creutz, S. E., Lotito, K. J., Fu, G. C. \& Peters, J. C. Photoinduced Ullmann $\mathrm{C}-\mathrm{N}$ coupling: demonstrating the viability of a radical pathway. Science 338, 647-651 (2012).

(53) Yoo, W.-J., Tsukamoto, T. \& Kobayashi, S. Visible light-mediated Ullmann-type C-N coupling reactions of carbazole derivatives and aryl iodides. Org. Lett. 17, 3640-3642 (2015).

(54) Hwang, S. J. et al. Trap-free halogen photoelimination from mononuclear Ni(III) complexes. $J$. Am. Chem. Soc. 137, 6472-6475 (2015).

(55) Shields, B. J. \& Doyle, A. G. Direct C $\left(s p^{3}\right)-$ H cross coupling enabled by catalytic generation of chlorine radicals. J. Am. Chem. Soc. 138, 12719-12722 (2016).

(56) Heitz, D. R., Tellis, J. C. \& Molander, G. A. Photochemical nickel-catalyzed C-H arylation: synthetic scope and mechanistic investigations. J. Am. Chem. Soc. 138, 12715-12718 (2016). 
(57) Dumele, O., Wu, D., Trapp, N., Goroff, N. \& Diederich, F. Halogen bonding of (iodoethynyl)benzene derivatives in solution. Org. Lett. 16, 4722-4725 (2014).

\section{Acknowledgments}

We thank Sorbonne Université, CNRS and ANR HyperSiLight for funding and Chinese Scholarship Council (Ph.D. grants to Z.X. and F.Z.). We are grateful to Omar Khaled for HRMS. This work was granted access to the HPC resources of the HPCaVe centre at Sorbonne Universite and the authors wish to acknowledge the support from the ICMG Chemistry Nanobio Platform-PCECIC, Grenoble, for calculations facilities. Jérémy Forté is acknowledged for the XRD analyses.

\section{Authors contributions}

Z.X., V.C. and F.Z. performed the synthetic experiments and did all the physicochemical analyses. C.P. did the MS analyses. A.E., L.J. and T.L.S. performed and analyzed the luminescence and transient absorption experiments. Y.G. and H.D. carried out computational studies and V.M-M., C.O., L.F. designed the experiments, collated the data and prepared the manuscript.

\section{Competing Interests}

The authors declare no competing interests.

\section{Additional information}

Correspondence and request for materials should be addressed to V.M.-M., C.O. or L.F.

\section{List of the figure captions}

Fig 1. Gold-catalyzed additions to alkynes. a General scheme of the dual gold and photoredox catalyzed difunctionalization of alkynes. b Proposed mechanistic pathway I in dual gold-photoredox catalysis. The key $\mathrm{Au}(\mathrm{III})$ intermediate $\mathbf{B}$ is generated via radical addition on the $\mathrm{Au}(\mathrm{I})$ precatalyst and the resulting $\mathrm{Au}(\mathrm{II})$ complex $\mathbf{A}$ is oxidized through SET by the oxidized photocatalyst. Pathway I has been supported by calculations and the isolation of intermediates. c. Alternate mechanistic pathway II implies the initial formation of a vinyl $\mathrm{Au}(\mathrm{I})$ intermediate $\mathbf{E}$. d This work: dual gold and photoinduced alkynylative $O$-cyclization via photosensitized oxidative addition.

Fig. 2 A vinylgold(I) as plausible intermediate. a Preparation of vinylgold(I) 6 according to Hashmi's method. b XRD structure of complex 6. c Alkynylation from $\mathbf{2 b}$ and $\mathbf{6}$ under blue LED irradiation. $\mathbf{d}$ Alkynylation of $\mathbf{6}$ by $\mathbf{2} \mathbf{b}$ by direct irradiation and sensitization. e Quenching of ${ }^{3}$ [ $\mathbf{I r}-\mathbf{F}$ ] by vinylgold 6 monitored by steady-state fluorimetry. f Differential absorption spectra of ${ }^{3}$ [Ir-F] (500 $\mu \mathrm{M}$ in $\mathrm{N}_{2}$-saturated acetonitrile) in the presence of increasing amounts of vinylgold 6 showing the quenching of the absorption of ${ }^{\mathbf{3}}[\mathbf{I r}-\mathbf{F}]$ at $500 \mathrm{~nm}$ and the rise of new contributions assigned to ${ }^{\mathbf{3}} \mathbf{6}$ below $450 \mathrm{~nm}$ and above $550 \mathrm{~nm}$. Spin density isosurface (isovalue 0.0006 a.u.) g isolated ${ }^{3}$ [Ir-F] complex. $\mathbf{h}^{3}[\mathbf{I r}-\mathbf{F}]$ in the vicinity of $\mathbf{6}$.

Fig. 3. Potential energy surface of the reaction of ${ }^{3} 6$ with $2 a$. The reaction of ${ }^{\mathbf{3}} \mathbf{6}$ with $\mathbf{2 a a}$ proceeds via several steps to lead to intermediate ${ }^{3} \mathbf{I V}$. Final formation of $\mathbf{3 a a}$ follows either the dissociative decay of ${ }^{\mathbf{3}} \mathbf{I V}$ or occurs after rearrangement and dissociation of ${ }^{\mathbf{3}} \mathbf{I V}$ into ${ }^{\mathbf{3}} \mathbf{3} \mathbf{a a}$. Optimized geometries of intermediates ${ }^{3} \mathbf{I I}$ and ${ }^{3} \mathbf{I V}$ are given in the top-right insert of the figure. Salient feature of complex ${ }^{3}$ II are the I-C-C angle of $115^{\circ}$ and a C-Au bond on $2.12 \AA$. Gibbs free energies $\left(\mathrm{CH}_{3} \mathrm{CN}\right)$ are given relatively to the starting products and are in $\mathrm{kcal} / \mathrm{mol}$. 
Fig 4. Mechanism proposal. Two intertwined catalytic cycles are responsible for the alkynylative cyclization of $o$-alkylnylphenols with iodoalkynes. The connecting point involve the interaction of ${ }^{3}[\mathbf{I r}-\mathbf{F}]$ with vinylgold $\mathbf{6}$ to promote the formation of ${ }^{\mathbf{3}} \mathbf{6}$ prone to undergo oxidative addition.

Fig. 5. Scope of the alkynylation process. a Variation of the iodoalkynes $\mathbf{2}$. b Variation of the $o$ alkynylphenols 1. c Post-functionalization of benzofuran 3aa by stereoselective hydrogenation and [3+2] cycloaddition. d Other working systems involving other nucleophilic and electrophilic partners. Experimental details: a 5 mol \% [AuCF $\mathbf{C}$ ], 1 mol\% [Ir-F], $\mathrm{K}_{2} \mathrm{CO}_{3}$ (2.5 equiv), 1,10phenanthroline (10 mol\%) MeCN, blue LED, r.t., overnight; ${ }^{\text {b }} 1$ mol\% [Ir-F], 1,10-phenanthroline (10 mol\%) MeCN, blue LED, r.t., overnight. ${ }^{c}$ NMR yield.

Table 1. Defining the key parameters of the alkynative cyclization ${ }^{\mathrm{a}}$

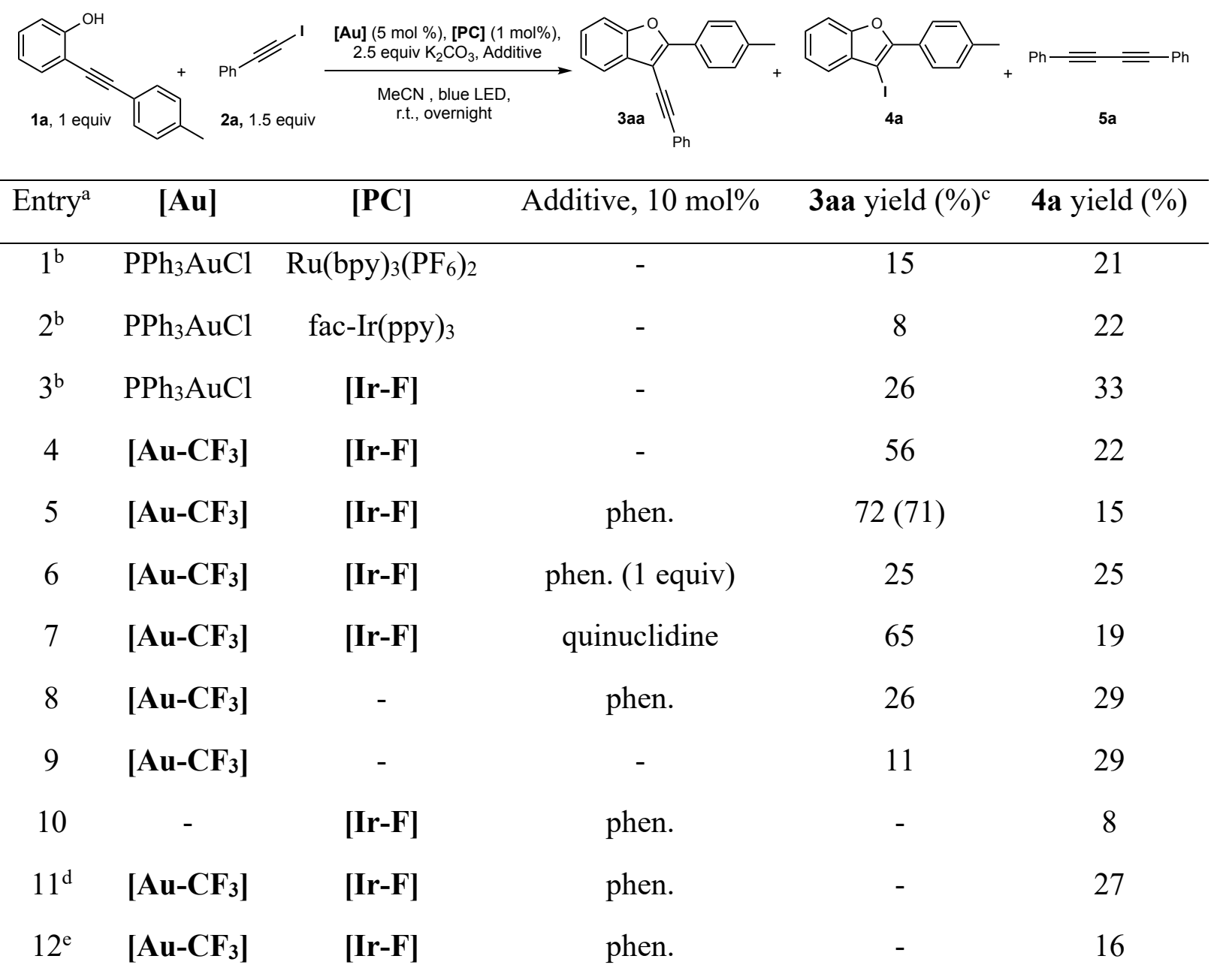

${ }^{\mathrm{a}}\left[\mathbf{A u}-\mathbf{C F}_{3}\right]=\left(p-\mathrm{CF}_{3} \mathrm{Ph}\right)_{3} \mathrm{PAuCl} ;[\mathbf{I r}-\mathbf{F}]=\operatorname{Ir}\left[\mathrm{dF}\left(\mathrm{CF}_{3}\right) \text { ppy }\right]_{2}(\mathrm{dtbbpy}) \mathrm{PF}_{6} ;$ phen. $=1,10$-phenanthroline. ${ }^{\mathrm{b}}$ Only 1 equiv of $\mathrm{K}_{2} \mathrm{CO}_{3}$ was used. ${ }^{\mathrm{c}}$ Yields are determined by ${ }^{1} \mathrm{H}$ NMR using 1,3,5-trimethoxybenzene as internal standard, yield in parentheses is isolated yield. ${ }^{\mathrm{d}} \mathrm{No} \mathrm{K}_{2} \mathrm{CO}_{3} .{ }^{\mathrm{e}}$ No light.

\section{Table of contents summary}

Iridium photosensitization triggers oxidative addition at organogold intermediates. This undescribed process in gold coordination tunnels the difficult oxidative addition - reductive elimination sequence 
and allows a novel dual catalysis to promote Csp2-Csp and Csp2-Csp2 cross coupling reactions. It also opens important prospects in the field of excited state gold catalysis. 